\title{
Questionário de Bem-Estar no Trabalho: estrutura e propriedades psicométricas
}

\author{
Questionnaire of Well-Being at Work: structure \\ and psychometric properties
}

\author{
Patrícia Martins GOULART \\ Josep Maria Ribas BLANCH ${ }^{2}$ \\ Miguel Angel SAHAGÚN² \\ Tamara Sarate BOBSIN ${ }^{1}$
}

\begin{abstract}
Resumo
Este artigo tem como objetivo apresentar a estrutura e as propriedades psicométricas da versão brasileira do Questionário de Bem-Estar no Trabalho. Essa ferramenta foi projetada para uma medição particularmente sensível aos aspectos psicossociais do bem-estar no trabalho, bem como à natureza bipolar dessa experiência de bem-estar. O questionário foi aplicado a uma amostra constituída por 101 docentes de uma instituição comunitária de ensino superior no sul catarinense. A análise fatorial mostra dois fatores independentes no Questionário Bem-Estar no Trabalho, o primeiro considerando três escalas de bem-estar psicossocial (afetos, competências e expectativas), e o segundo levando em conta três escalas de efeitos colaterais (somatização, desgaste e alienação). As escalas mostraram uma alta consistência interna, com alfas de Cronbach de 95, 95, 97, 88, 93 e 80, respectivamente. O Questionário Bem-Estar no Trabalho reproduz fielmente a estrutura do modelo teórico assumido e reúne as propriedades técnicas necessárias para avaliar o bem-estar no trabalho em ambientes universitários.
\end{abstract}

Unitermos: Bem-estar psicossocial. Questionários. Trabalho. Validação de teste.

\begin{abstract}
The objective of this article is to present the structure and psychometric properties of the Brazilian version of The Work Well-Being Questionnaire. This was developed with the aim of providing a measurement tool particularly sensitive to the psychosocial dimension of work well-being, and to the bipolar nature of the well-being experience. The questionnaire was applied to a convenience and intentional sample, constituted by 101 teachers of a higher education community institution in the south of Santa Catarina. Factor analysis shows two independent factors in The Work Well-Being Questionnaire, considering the first of threescales of psychosocial well-being (emotions, skills and expectations) and the second, three other side effects (somatization, wear and alienation). The scales showed high internal consistency with Cronbach's alphas of 95, 95, 97, 88, 93 and 80, respectively. The Work Well-Being Questionnaire faithfully reproduces the structure of the theoretical model assumed and meets the technical properties needed to assess the work well-being in university environments.
\end{abstract}

Uniterms: Psychosocial well-being. Questionnaires. Work. Validation Test validity.

$\cot$

1 Universidade do Extremo Sul Catarinense, Unidade Acadêmica de Ciências Sociais Aplicadas, Faculdade de Psicologia. Av. Universitária, 1105, Universitário, 88806-000, Criciúma, SP, Brasil. Correspondência para/Correspondence to: P.M. GOULART. E-mail: <pmg@unesc.net>.

2 Universidad Autònoma de Barcelona, Facultad de Psicología, Departamento de Psicología Social. Barcelona, España. Apoio: Ministerio de Educación y Ciencia SEJ2007-63686/PSIC. 
O propósito deste estudo é tornar público o caminho trilhado no processo de validação, no contexto brasileiro, do instrumento espanhol Questionário de Bem-Estar no Trabalho (qBET), apresentando sua estrutura e propriedades psicométricas, concebidas para avaliar a dimensão psicossocial do bem-estar no trabalho.

O bem-estar deriva de um conjunto de juízos valorativos e de reações emocionais concernentes ao grau de satisfação, prazer e felicidade em que a própria experiência é vivida (Diener, 2000; Diener \& Suh, 2001; Diener, Oishi \& Lucas, 2003). A Organização Mundial da Saúde, em sua carta de constituição de 1948, situa o conceito no mesmo núcleo da definição da saúde, como estado de completo bem-estar físico, psicológico e social. A literatura da Psicologia Social do Trabalho, por sua vez, invoca os termos bem-estar subjetivo (Diener, 2000) e bem-estar psicológico (Ryff \& Keyes, 1995), relacionando-os ao campo semântico da felicidade, saúde, satisfação e qualidade de vida (Avia \& Vázquez, 1998; Blanco \& Díaz, 2005; Csikszentmihalyi, 1990; Hills \& Argyle, 2002; Seligman, 2002; Seligman \& Csikszentmihalyi, 2000).

Em uma visão retrospectiva, o mal-estar, o sofrimento e as doenças formam o núcleo das preocupações teóricas e das pesquisas empíricas sobre a experiência do trabalho. Schaufeli e Backer (2004) referem que, no início do século XXI, as investigações psicológicas sobre aspectos negativos da experiência laboral (distresse, burnout, ansiedade, depressão, transtornos mentais menores, disfunções psicofisiológicas etc.) predominaram com uma porcentagem de 15 a 1 sobre as que trataram de suas facetas positivas (felicidade, satisfação, bem-estar, autorrealização etc.). Nesse caso, a ênfase recai sobre sintomas individuais que aparecem em determinados contextos organizacionais e condições do trabalho.

Dentre as ferramentas mais frequentemente utilizadas para avaliar o bem-estar no trabalho, destacam-se instrumentos clínicos como o General Health Questionnaire (GHQ, desenhado por Goldberg nos anos setenta), que enfoca especialmente transtornos psíquicos menores, como insônia, depressão e hipocondria. No Brasil, esse Questionário de Saúde Geral, na sua versão de 12 Itens (QSG-12), validado por Gouveia, 658 Barbosa, Andrade e Carneiro (2010) tem sido ampla- mente usado. Também, utilizam-se os inventários e escalas (como o Inventário de Depressão de Beck-BDI, o Inventário de Ansiedade de Beck-BAl e a Escala de Desesperança de Beck-BHS) para avaliar efeitos como depressão, ansiedade e ideação suicida, embora suas normas de utilização tenham sido desenvolvidas para uso com pacientes psiquiátricos (Cunha, 2001).

No que se refere a instrumentos desenhados especificamente para a avaliação de aspectos relacionados ao bem-estar no trabalho, um dos mais utilizados é o Maslach Burnout Inventory, em suas diversas versões e adaptações em inglês (Maslach, Jackson \& Leiter, 1996), espanhol (Bresó, Salanova, Schaufeli \& Nogareda, 2007) e português brasileiro (Carlotto \& Câmara, 2008).

Em contraste com o enfoque predominantemente patologista, a corrente da Psicologia Positiva, que se conecta com os Current Concepts of Positive Mental Health de Jahoda (1958), propõe uma perspectiva centrada na avaliação da vertente positiva de bem-estar pessoal e laboral (Salanova \& Schaufeli, 2009; Seligman, 2002; Seligman \& Csikszentmihalyi, 2000; Warr, 2007), que se faz visível no Brasil por meio de estudos de Pasareli e Silva (2007). Entre os instrumentos representativos dessa orientação figura a Escala de Satisfação com a Vida, validada no Brasil por Albuquerque, Souza e Martins (2010). Segue nessa linha o Oxford Happiness Questionnaire (Hills \& Argyle, 2002), o Quality of Life Enjoyment and Life Satisfaction Questionnaire (Endicott, Nee, Harrison \& Blumenthal, 1993) e as Escalas de Bem-Estar Psicológico de Ryff (Ryff \& Keyes, 1995; Van Dierendonck, 2004; Díaz et al., 2006), esta última utilizada no Brasil por Covacs (2006). Por fim, pode-se apontar a Escala de Bienestar Psicológico de Sánchez-Cánovas (1998). A investigação sobre qualidade de vida mediante indicadores sociais aporta também instrumentos de medida do bem-estar no trabalho com ferramentas para avaliação geral do Selfreported well-being (Andrews \& McKennell, 1980), da Quality of Well-being da Happy Life Expectancy (Veenhoven, 1996) ou do Happy Planet Index (HPI), que inclui aspectos econômicos e ecológicos do bem-estar humano (Abdallah, Thompson, Michaelson, Marks \& Steuer, 2009; Marks Aldallah, Simms \& Thompson, 2006). O World Health Organization Quality of Life Assessment Instrument (WHOQOL-100), inclui também medidas específicas de bem-estar concernentes às relações sociais. 
No Brasil, o Grupo de Qualidade de Vida da Divisão de Saúde Mental da Organização Mundial de Saúde (WHOQOL Group, 1994) baseia-se nos pressupostos de que qualidade de vida é um construto subjetivo (percepção do indivíduo em questão), multidimensional e composto por dimensões positivas (por exemplo, mobilidade) e negativas (por exemplo, a dor). Observa-se que o Work-Related Quality of Life Scale (WRQoWL) (Van Laar, Edwards \& Easton, 2007; Edwards, Van Loar \& Easton, 2009) mede alguns fatores do bem-estar no trabalho.

Para a configuração específica do qBET aplicaram-se dois critérios: (a) integração da pluralidade dos abordagens tradicionais do objeto e (b) superação do duplo viés patologista e individualista dessa tradição, utilizando escalas bipolares para avaliar tanto o polo negativo (o mal-estar) quanto o positivo (bem-estar), com conteúdos que compõem os fatores individuais e organizacionais do bem-estar no trabalho.

Com o propósito de colaborar nessa trajetória, este estudo tem por objetivo apresentar a estrutura e as propriedades psicométricas do qBET, concebido como ferramenta específica de avaliação da dimensão psicossocial e do caráter bipolar (positivo versus negativo) dessa experiência. Diferentemente da maioria das escalas de bem-estar citadas, que se referem a dimensões gerais da vida e da saúde, com o qBET pretende-se avaliar o bem-estar psicológico situado no trabalho.

\section{Método}

O primeiro passo consistiu na tradução do instrumento original. A tradução da língua espanhola para a língua portuguesa (brasileira) contou com a avaliação de um grupo de juízes e o pré-teste da versão final. Seguindo um procedimento adotado em estudos anteriores, participaram dois tradutores independentes, um dos quais tem o português como língua materna, enquanto o outro é de origem espanhola. Cada qual realizou sua versão do instrumento, sendo ambas avaliadas individualmente e depois comparadas entre si por dois outros pesquisadores em psicologia, para obtenção de uma única versão final em português.

Após os ajustes necessários, a versão final do instrumento foi submetida a um pré-teste para análise da compreensibilidade dos itens das escalas. Foi utilizada a técnica de prova, que consiste na determinação da compreensibilidade do instrumento a ser aplicado a uma amostra da população-alvo. Essa técnica permitiu avaliar a validade aparente (validade de conteúdo) do instrumento adaptado. Participaram do pré-teste seis sujeitos. Essa delimitação de participantes ateve-se à análise em profundidade das informações obtidas. As instruções sobre o preenchimento das escalas, assim como ao que se refere a cada um dos itens, foram discutidas com os participantes quanto à compreensão e à clareza, e levantadas as discrepâncias sobre a compreensão geral do instrumento. As escalas, aplicadas em forma de questionário, foram respondidas de modo individual, com registros sobre dúvidas e comentários gerais para refinamento do instrumento.

\section{Participantes}

Responderam ao questionário 101 pessoas, sendo 54,5\% mulheres e 45,5\% homens, todos professores de uma universidade sem fins lucrativos. A idade média dos homens foi de 43 anos (Desvio-Padrão-DP=8,63), e a das mulheres, $42(D P=7,97)$. Dentre os critérios de inclusão destacam-se a heterogeneidade dos participantes quanto à formação acadêmica original, tempo de atuação profissional e titulação, bem como a simetria na representação por gênero.

\section{Instrumento}

Uma versão preliminar e menos extensa do instrumento foi já aplicada numa investigação ibero-americana sobre qualidade de vida no trabalho a mais de 2200 pessoas em diferentes tipos de situação ocupacional (Blanch, 2005; 2006). Uma parte da versão foi concretamente desenvolvida no Brasil (Goulart, 2005; 2007). O foco dessa pesquisa foi a experiência de trabalho no contexto de subemprego. As diversas escalas do instrumento mostraram boas propriedades psicométricas (com alfas compreendidos entre 0,84 e 0,95). Posteriormente, uma nova versão do questionário foi construída para a avaliação da experiência do trabalho de (1 200) profissionais qualificados que trabalham nas áreas do ensino superior e dos serviços de saúde (Blanch, 2009; Blanch, Sahagún, Cantera \& Cervantes, 2010).

O modelo teórico sobre bem-estar no trabalho que orientou o desenho desse questionário inclui duas 
dimensões principais, Bem-Estar Psicossocial (com os domínios afetos, competências e expectativas) e Efeitos Colaterais (com os domínios somatização, desgaste e alienação), organizadas em seis escalas (Figura 1). Os itens de algumas escalas foram redigidos com formato de diferencial semântico, enquanto para as outras adotou-se um modelo Likert.

O primeiro passo do desenho de cada uma das escalas do questionário foi a seleção de itens com critérios de relevância, partindo de uma revisão da literatura, que permitiu identificar os tópicos centrais da investigação sobre o problema. Depois, atendeu-se ao princípio psicométrico da representatividade teórica (Álvaro, 1997; Camarero, 2004). Este último critério permite valorar a medida em que as dimensões e indicadores específicos do Questionário representam aspectos importantes da experiência do bem-estar laboral do coletivo investigado. Para isso, procedeu-se a uma revisão piloto por uma comissão integrada por pessoas do setor profissional implicado.

Haja vista o qBET remeter especificamente ao bem-estar psicológico no trabalho, as instruções de aplicação e o enunciado de cada escala remete direta e explicitamente à própria circunstância laboral. Assim, para responder às duas primeiras perguntas, a pessoa deve-se colocar na seguinte situação: Atualmente, em meu trabalho, sinto... O mesmo acontece com a terceira: Em minha trajetória laboral... . E com as outras três restantes: Atualmente, por causa de meu trabalho, sinto.... O conjunto do Questionário, que inclui também um bloco referente a Condiçoes de Trabalho (Blanch, Sahagún \& Cervantes, 2010), levanta questões abertas para uma aproximação qualitativa, que permite comparar a informação numérica e a textual sobre o mesmo objeto do estudo. Entre elas, figura a eleição de quatro palavras-chave que definem a experiência de trabalho atual e a caracterização e avaliação das mudanças percebidas nas condições (materiais, sociais, técnicas, financeiras, contratuais, temporais, organizacionais etc.) do própio trabalho.

\section{Procedimento}

O tempo médio de aplicação do questionário geral variou entre 45 e 60 minutos. Devido às características do público-alvo investigado, supostamente com pouco tempo disponível, não se estimou acessar aleatoriamente as pessoas participantes, por previsível baixa porcentagem de respostas. Tampouco se fez de cima para baixo (buscando a cumplicidade da direção da universidade), para garantir as máximas cotas possíveis

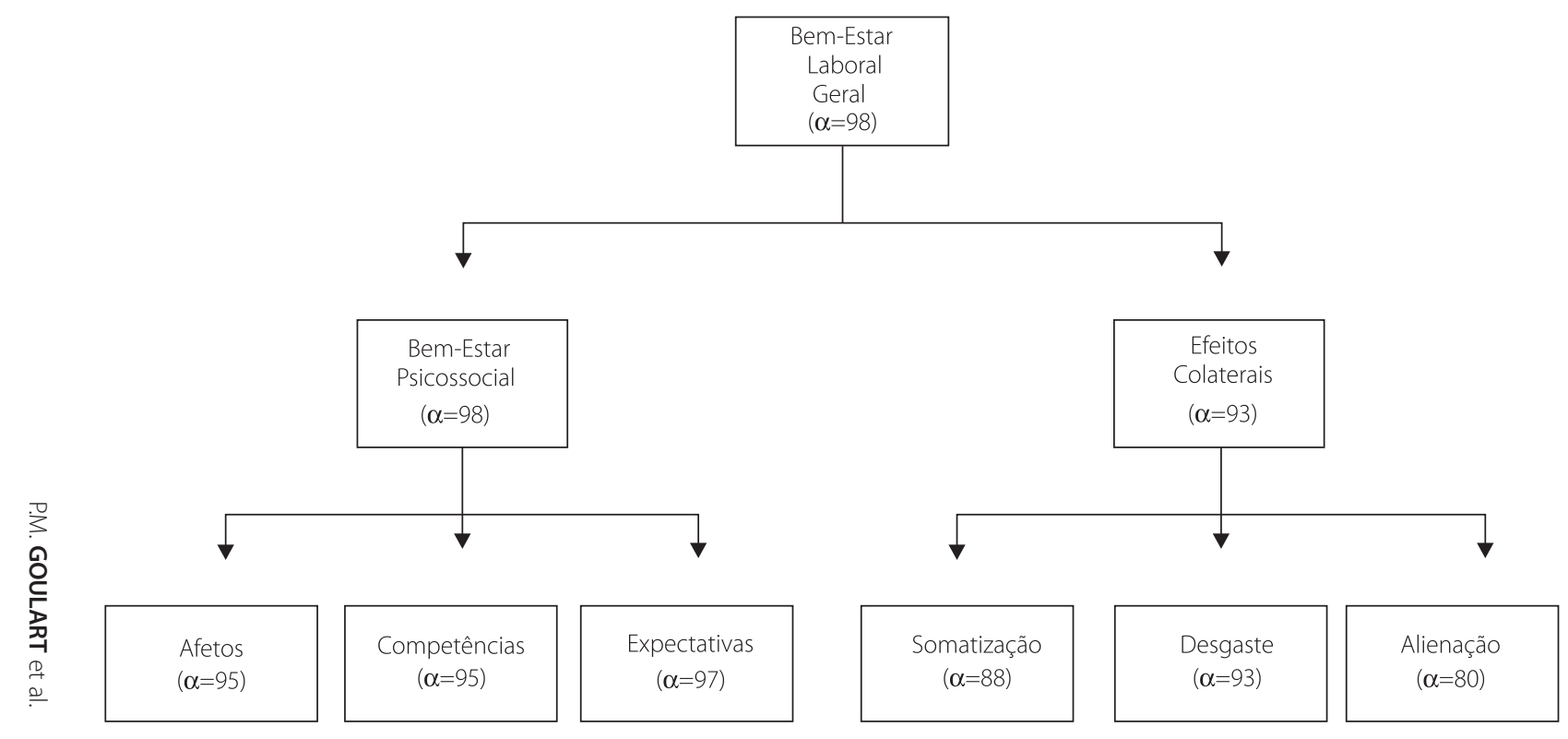

Figura 1. Coeficientes alfa do Questionário, fatores e escalas do qBET.

660

Nota: qBet: Questionário de Bem-Estar no Trabalho. 
de credibilidade, confiabilidade e anonimato. Estabeleceu-se que seriam entrevistados docentes de diferentes cursos, a fim de garantir maior representatividade de respostas. Assim, o questionário foi aplicado de modo individualizado, com apoio de acadêmicos, os quais faziam o agendamento prévio com os professores, que respondiam ao questionário nas dependências da universidade.

Para o levantamento psicométrico do questionário, foram realizadas as seguintes análises: (a) de itens, mediante índice de homogeneidade (correlação item/fator), considerando dois fatores gerais e seis fatores específicos (escalas); (b) de fidedignidade, identificando o coeficiente alfa de Cronbach de cada um dos mencionados fatores gerais e específicos, assim como do mesmo questionário global; (c) de validez de conteúdo, por revisão de literatura e consenso de juízos e (d) de validez de construto, com análises fatoriais exploratórias utilizando o método de componentes principais, e validez de critério, medindo correlações com outras escalas. Aplicou-se outra análise fatorial de componentes principais com rotação promax para as séries de itens integrados em cada um dos fatores gerais, aparecendo três escalas em cada fator, tal como propõe o modelo teórico seguido.

Em todas as fases, os procedimentos de aplicação adotados envolveram o cumprimento das diretrizes da Resolução do Conselho Nacional de Saúde (CNS) 196/96, referentes a pesquisa envolvendo seres humanos, a citar a liberdade de participação e o conhecimento da pesquisa através de Termo de Consentimento Livre e Esclarecido e demais preceitos contidos na referida Resolução.

\section{Resultados}

Na Figura 1 apresenta-se o modelo geral, no qual são incluídas as pontuações alfa de Cronbach, obtidas para o conjunto do questionário, assim como para cada um de seus fatores e escalas. Os valores alfa variam entre 0,80, correspondente à escala com menor número de itens (4), e 0,97, correspondente à escala com maior número dos mesmos (22).

Na Figura 2 apresenta-se o gráfico de sedimentação das escalas de bem-estar psicossocial e, na Tabela 1, a estrutura fatorial das mesmas, obtidas mediante

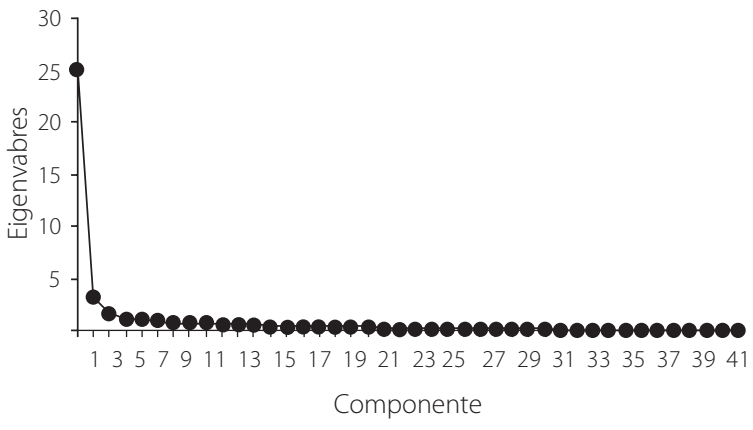

Figura 2. Sedimentação de componentes principais das Escalas de Bem-Estar Psicossocial.

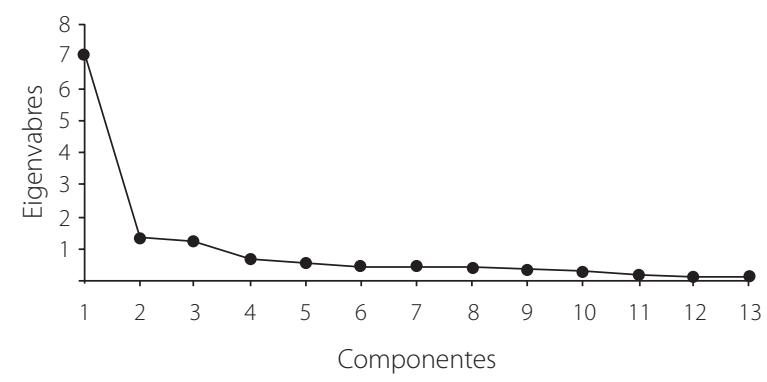

Figura 3. Sedimentação de componentes principais das Escalas de Efeitos Colaterais.

rotação promax, assim como os autovalores iniciais e a variância explicada.

A Figura 3 apresenta o gráfico de sedimentação das escalas incluídas no fator geral de efeitos colaterais, enquanto que a Tabela 2 mostra, por meio do mesmo procedimento de rotação, a estrutura fatorial das escalas desse fator, os autovalores iniciais e a variância explicada.

O fator geral 1 (bem-estar psicossocial) está composto por 42 itens, agrupados em três subfatores: as escalas de Afetos (10 itens), de Competências (10 itens) e de Expectativas (22 itens). De outro lado, compõem o fator geral 2 (Efeitos colaterais) três subfatores: as escalas de Somatização (5 itens), de Desgaste (4 itens) e de Alienação (4 itens).

A análise fatorial exploratória do questionário Bem-estar Laboral Geral (BLG), utilizando o método de componentes principais, com rotação varimax, evidenciou os fatores gerais com autovalores superiores a 1, que coincidem com as duas dimensões do bem-estar laboral propostas pelo modelo teórico que orientou a investigação e que explicam em conjunto os 83\% da variância total. A Tabela 2 mostra essa estrutura fatorial. 
Tabela 1. Estrutura Fatorial das Escalas de Bem-Estar Psicossocial. Criciúma (SC), 2009.

\begin{tabular}{|c|c|c|c|}
\hline \multirow{2}{*}{ Itens das escalas } & \multicolumn{3}{|c|}{ Componentes } \\
\hline & 1 & 2 & 3 \\
\hline Insatisfação - Satisfação & 0,714 & 0,652 & 0,830 \\
\hline Insegurança - Segurança & 0,623 & 0,581 & 0,862 \\
\hline Intranquilidade - Tranquilidade & 0,632 & 0,605 & 0,827 \\
\hline Impotência - Potência & 0,647 & 0,663 & 0,802 \\
\hline Mal-estar - Bem-estar & 0,610 & 0,645 & 0,801 \\
\hline Desconfiança - Confiança & 0,640 & 0,639 & 0,897 \\
\hline Insensibilidade - Sensibilidade & 0,729 & 0,622 & 0,850 \\
\hline Irracionalidade - Racionalidade & 0,732 & 0,797 & 0,604 \\
\hline Incompetência - Competência & 0,568 & 0,858 & 0,605 \\
\hline Imoralidade - Moralidade & 0,670 & 0,708 & 0,613 \\
\hline Maldade - Bondade & 0,448 & 0,750 & 0,697 \\
\hline Incerteza - Certeza & 0,612 & 0,711 & 0,849 \\
\hline Confusão - Clareza & 0,553 & 0,859 & 0,728 \\
\hline Desesperança - Esperança & 0,597 & 0,864 & 0,677 \\
\hline Fracasso - Êxito & 0,477 & 0,874 & 0,699 \\
\hline Dificuldade - Facilidade & 0,505 & 0,696 & 0,744 \\
\hline Incapacidade - Capacidade & 0,421 & 0,897 & 0,614 \\
\hline Pessimismo - Otimismo & 0,668 & 0,906 & 0,720 \\
\hline Ineficácia - Eficácia & 0,477 & 0,932 & 0,576 \\
\hline Inutilidade - Utilidade & 0,453 & 0,793 & 0,472 \\
\hline Minha motivação para o trabalho & 0,839 & 0,454 & 0,714 \\
\hline Minha identificação com os valores da organização & 0,849 & 0,579 & 0,739 \\
\hline Meu rendimento profissional & 0,815 & 0,539 & 0,568 \\
\hline Minha capacidade de gestão de minha carga de trabalho & 0,858 & 0,432 & 0,600 \\
\hline A qualidade das minhas condições de trabalho & 0,827 & 0,578 & 0,669 \\
\hline Minha autoestima profissional & 0,889 & 0,442 & 0,675 \\
\hline A cordialidade em meu ambiente social de trabalho & 0,693 & 0,635 & 0,690 \\
\hline A conciliação de meu trabalho com minha vida privada & 0,798 & 0,475 & 0,613 \\
\hline Minha confiança em meu futuro profissional & 0,807 & 0,545 & 0,717 \\
\hline Minha qualidade de vida laboral & 0,724 & 0,719 & 0,757 \\
\hline O sentido do meu trabalho & 0,899 & 0,514 & 0,772 \\
\hline Mi acatamiento de las pautas de la dirección & 0,774 & 0,586 & 0,749 \\
\hline Meu estado de ânimo no trabalho/laboral & 0,827 & 0,678 & 0,798 \\
\hline Minhas oportunidades de promoção laboral & 0,782 & 0,569 & 0,783 \\
\hline Minha sensação de segurança no trabalho & 0,760 & 0,548 & 0,821 \\
\hline Minha participação nas decisões da organização. & 0,762 & 0,524 & 0,770 \\
\hline Minha satisfação com o trabalho & 0,884 & 0,480 & 0,817 \\
\hline Minha realização profissional & 0,882 & 0,507 & 0,789 \\
\hline O nível de excelência de minha organização & 0,748 & 0,471 & 0,582 \\
\hline Minha eficácia profissional & 0,882 & 0,483 & 0,613 \\
\hline Meu compromisso com o trabalho & 0,837 & 0,473 & 0,534 \\
\hline Minhas competências profissionais & 0,776 & 0,420 & 0,428 \\
\hline Autovalores iniciais & 25694 & 3510 & 1429 \\
\hline \% de Variância & 61175 & 8358 & 3402 \\
\hline \% Variância acumulada & 61175 & 69533 & 72935 \\
\hline
\end{tabular}

Rotação Promax.

\section{Discussão}

Os conteúdos temáticos do qBET estão enlaçados e concordam com aqueles da tradição clínica da psicopatologia do mal-estar no trabalho (Maslach

662 et al., 1996), como também com aqueles da so- ciologia da qualidade de vida (Edwards et al., 2009) e com estudos contemporâneos sobre a felicidade (Diener, 2000; Warr, 2007), mencionados na revisão introdutória. O qBET atendeu também aos critérios de equivalência semântica, idiomática e conceitual. 
Tabela 2. Estrutura Fatorial das Escalas de Efeitos Colaterais e Bem-Estar Laboral. Criciúma (SC), 2009.

\begin{tabular}{|c|c|c|c|}
\hline \multirow{2}{*}{ Escalas de Efeitos Colaterais } & \multicolumn{3}{|c|}{ Componentes } \\
\hline & 1 & 2 & 3 \\
\hline \multicolumn{4}{|l|}{ Itens das escalas } \\
\hline Sobrecarga de atividade laboral & 0,843 & 0,527 & 0,343 \\
\hline Desgaste emocional & 0,919 & 0,683 & 0,551 \\
\hline Esgotamento físico & 0,927 & 0,611 & 0,406 \\
\hline Saturação mental & 0,940 & 0,605 & 0,487 \\
\hline Mau humor & 0,737 & 0,603 & 0,683 \\
\hline Baixa realização profissional & 0,372 & 0,453 & 0,839 \\
\hline Trato despersonalizado & 0,313 & 0,209 & 0,746 \\
\hline Frustração & 0,492 & 0,585 & 0,813 \\
\hline Transtornos digestivos & 0,531 & 0,829 & 0,516 \\
\hline Dores de cabeça & 0,548 & 0,835 & 0,480 \\
\hline Insônia & 0,448 & 0,718 & 0,537 \\
\hline Dores nas costas & 0,586 & 0,885 & 0,287 \\
\hline Tensões musculares & 0,663 & 0,868 & 0,308 \\
\hline Autovalores iniciais & 7108 & 1371 & 1136 \\
\hline \% de Variância & 54677 & 10547 & 8741 \\
\hline \% Variância acumulada & 54677 & 65224 & 73966 \\
\hline \multicolumn{4}{|l|}{ Rotação Promax. } \\
\hline \multirow{2}{*}{ Questionário de Bem-Estar Laboral Geral } & \multicolumn{3}{|c|}{ Componentes } \\
\hline & 1 & & 2 \\
\hline \multicolumn{4}{|l|}{ Escalas } \\
\hline Afetos & \multicolumn{2}{|l|}{0,883} & 0,359 \\
\hline Competências & \multicolumn{2}{|l|}{0,842} & 0,382 \\
\hline Expectativas & \multicolumn{2}{|l|}{0,862} & 0,326 \\
\hline Somatização & \multicolumn{2}{|l|}{$-0,701$} & 0,566 \\
\hline Desgaste & \multicolumn{2}{|l|}{$-0,739$} & 0,508 \\
\hline Alienação & $-0,836$ & & 0,176 \\
\hline Autovalores iniciais & 3968 & & 990 \\
\hline \% de Variância & 66128 & & 16496 \\
\hline \% Variância acumulada & 66128 & & $\begin{array}{l}16496 \\
82624\end{array}$ \\
\hline
\end{tabular}

Rotação Varimax

De um ponto de vista metodológico, os resultados obtidos indicam que a estrutura do questionário reproduz fielmente o modelo teórico utilizado como base para essa investigação, representado na Figura 1. Os resultados mostram, ainda, que o instrumento reúne condições satisfatórias no que se refere à fidedignidade e validez, que a consistência interna das escalas é alta e que o processo de sua construção mostrou-se adequado aos padrões metodológicos. Blanch et al. (2010) observaram que o conjunto das escalas do fator bem-estar psicossocial apresentou correlações altas e significativas com a escala de satisfação com a organização (Blanch, 2009), que também formou parte desse macro questionário. Nessa comparação, a escala de Afetos apresentou uma correlação com a de Satisfação de 0,72 $(p<0,0001)$, a de Competências de 0,62 ( $p<0,0001)$ e a de Expectativas de $0,82(p<0,0001)$. Por outro lado, outra mostra de 182 pessoas de características demográficas homogêneas com respeito às 1252 que responderam ao instrumento de Blanch et al. (2010) preencheram esse mesmo questionário conjuntamente com a versão espanhola do Inventário de Burnout de Maslach (MBI) elaborada por Bresó et al. (2007). Na comparação das pontuações entre as diversas escalas, observou-se que as de fator efeitos colaterais correlacionaram-se positivamente com os fatores de MBl, e que as de fator bem-estar psicossocial correlacionaram-se negativamente com eles. 


\section{Considerações Finais}

O Questionário do Bem-Estar no Trabalho corresponde à estrutura do modelo teórico que o fundamenta e possui excelentes propriedades psicométricas. Além disso, apresenta um duplo valor: (a) avalia especificamente o bem-estar laboralmente situado, atendendo às faces positiva e negativa dessa experiência e a seus fatores pessoais e organizacionais; e (b) foi produzido e validado no contexto ibero-americano e aplicado no âmbito específico de atividades profissionais de serviços a pessoas. Entre suas limitações consta o fato de que, neste momento, apenas permite obter uma informação descritiva. No que se refere a seu uso prático como ferramenta de diagnóstico, está-se trabalhando na construção de parâmetros que permitam estabelecer grupos de comparação por setores profissionais. A experiência permite dizer que o qBET é um instrumento de fácil aplicação, na medida em que requer instruções simples, com possibilidade de administração tanto individual quanto coletiva.

\section{Referências}

Abdallah, S., Thompson, S., Michaelson, J., Marks, N., \& Steuer, N. (2009). The Happy Planet Index 2.0. London: New Economics Foundation.

Albuquerque, J. B., Souza, F. M., \& Martins, C. R. (2010). Validação das escalas de satisfação com a vida e afetos para idosos rurais. Psico, 41 (1), 85-92.

Alvaro, M. (1997). Principios psicométricos de la evaluación psicológica. In G. Buela \& J. C. Sierra (Coords.), Manual de evaluación psicológica:fundamentos, técnicas y aplicaciones (pp.173-192). Madrid: Siglo XXI.

Andrews, F. M., \& McKennell, A. C. (1980). Measures of self reported well-being: their affective, cognitive and other components. Social Indicators Research, 8 (2), 127-155.

Avia, M. D., \& Vázquez, C. (1998). Optimismo inteligente. Madrid: Alianza.

Blanch, J. M. (2005). Estudio de calidad de vida laboral en el subempleo. In J. Romay, J. Salgado, M. Romaní \& D. Robla (Comps.), Psicología de las organizaciones, del trabajo y recursos humanos y de la salud (pp.79-87). Madrid: Biblioteca Nueva.

Blanch, J. M. (2006). Estudio internacional sobre la calidad de la experiencia laboral en el capitalismo flexible. In X. Díaz, L. Godoy, A. Stecher \& J. P. Toro (Coords.), Trabajo, identidad y vínculo social: reflexiones y experiencias en el capitalismo flexible (pp.75-107). Santiago de Chile: CEMUDP.

Blanch, J. M. (2009). Propiedades psicométricas de las escalas del cuestionario: estudio piloto. Documento interno de trabajo del grupo investigación Colaborando. Bellaterra: Universidad Autónoma de Barcelona.

Blanch, J. M., Sahagún, M., Cantera, L., \& Cervantes, G. (2010). Cuestionario de Bienestar Laboral General: estructura y propiedades psicométricas. Revista de Psicología del Trabajo y las Organizaciones, 26 (2), 157-170.

Blanch, J. M., Sahagún, M., \& Cervantes, G. (2010). Estructura factorial de la escala de condiciones de trabajo: factor structure of working conditions scale. Revista de Psicología del Trabajo y las Organizaciones, 26 (3), 175-189.

Blanco, A., \& Díaz, D. (2005). El bienestar social: su concepto y medición. Psicothema, 17 (3), 580-587.

Bresó, E., Salanova, M., Schaufeli, W. B., \& Nogareda, C. (2007). Síndrome de estar quemado por el trabajo "Burnout" (III): instrumento de medición. (Nota técnica de Prevención, 732, 21 a Serie). Madrid: INSHT.

Camarero, L. (2004). Representatividad estadística versus social. Metodología de Encuestas, 6 (1) 61-70.

Carlotto, M. S., \& Câmara, S. G. (2008). Síndrome de burnout e estratégias de enfrentamento em professores de escolas públicas e privadas. Psicologia da Educação, 26, 29-46.

Covacs, J. M. L. M. (2006). Bem-estar no trabalho: o impacto dos valores organizacionais, percepção de suporte organizacionale percepções de justiça. Dissertação de Mestrado não-publicada, Programa de Pós-Graduação em Psicologia da Saúde, Universidade Metodista de São Paulo. Recuperado em dezembro, 2011, disponível em <http:// ibict.metodista.br>.

Csikszentmihalyi, M. (1990). Flow: the psychology of optimal experience. New York: Harper and Row.

Cunha, J. A. (2001). Manual daversão em português das Escalas Beck. São Paulo: Casa do Psicólogo.

Díaz, D., Rodríguez-Carvajal, R., Blanco, A., Moreno-Jiménez, B; Gallardo I., Valle, C., et al. (2006). Adaptación española de las escalas de bienestar psicológico de Ryff. Psicothema, 18 (3), 572-577.

Diener, E. (2000). Subjective well-being: the science of happiness and a proposal for a national index. American Psychologist, 55 (1), 34-43.

Diener, E., Oishi, S., \& Lucas, R. E. (2003). Personality, culture, and subjective well-being: emotional and cognitive evaluations of life. Annual Review of Psychology, 54 (1), 403-425.

Diener, E., \& Suh, E. (2001). Culture and subjective well-being. Cambridge, MA: MIT Press.

Edwards, J., Van Laar, D. L., \& Easton, S. (2009). The WorkRelated Quality of Life (WRQoL) scale for higher education employees. Quality in Higher Education, 15 (3), 207-219.

Endicott, J., Nee, J., Harrison, W., \& Blumenthal, R. (1993). Quality of life enjoyment and life satisfaction questionnaire: a new measure. Psychopharmacological Bulletin, 28, 321-326.

Goulart, P. M. (2005). La experiencia de trabajar en un entorno flexible: el modelo brasileño. In J. Romay, J. Salgado, M. Romaní \& D. Robla. (Orgs.), Psicología de las 
organizaciones, y recursos humanosy de la salud. Psicología de las organizaciones, y recursos humanos y de la salud (Vol. 1, pp.89-93). Madrid: Biblioteca Nueva.

Goulart, P. M. (2007). Trabajar en el nuevo capitalismo. Bellaterra: Universitat Autònoma de Barcelona.

Gouveia, V. V., Barbosa, G. A., Andrade, E. O., \& Carneiro, M. B. (2010). Factorial validity and reliability of the General Health Questionnaire (GHQ-12) in the Brazilian physician population. Caderno de Saúde Pública, 26 (7), 1439-1445. Recuperado em dezembro, 2011, disponível em <http:// www.scielo.br>

Hills, P., \& Argyle, M. (2002). The Oxford Happiness Questionnaire: a compact scale for the measurement of psychological well-being. Personality and Individual Differences, 33 (7), 1073-1082.

Jahoda, M. (1958). Current concepts of positive mental health. New York: Basic Books.

Marks, N., Abdallah, S., Simms, A., \& Thompson, S. (2006). The happy planet index 1.0. London: New Economics Foundation.

Maslach, C., Jackson, S. E., \& Leiter, M. P. (1996). Maslach burnout inventory manual ( $3^{\text {rd }}$ ed.). Palo Alto: CA: Consulting Psychologists Press.

Passareli, P. M., \& Silva, J. A. (2007). Psicologia positiva e o estudo do bem-estar subjetivo. Estudos de Psicologia (Campinas), 24 (4), 513-517. doi: 10.1590/S0103-166X2 007000400010

Ryff, C., \& Keyes, C. (1995). The structure of psychological well-being revisited. Journal of Personality and Social Psychology, 69 (1), 719-727.

Salanova, M., \& Schaufeli, W. (2009). El engagement en el trabajo: cuando el trabajo se convierte en pasión. Madrid: Alianza.
Sánchez-Cánovas, J. (1998). Escala de bienestar psicológico. Madrid: TEA.

Seligman, M. E. P. (2002). Authentic Happiness: using the new positive psychology to realize your potential for lasting fulfillment. New York: Free Press.

Seligman, M. E. P., \& Csikszentmihalyi, M. (2000). Positive psychology: an introduction. American Psychologist, 55 (1), 5-14.

Schaufeli, W. B., \& Backer, A. B. (2004). Job demands, job resources, and their relationship with burnout and engagement:a multi-sample study. Journal of Organizational Behavior, 25 (3), 293-315.

Van Dierendonck, D. (2004). The construct validity of Ryff's Scale of Psychological well-being and its extension with spiritual well-being. Personality and Individual Differences, 36 (3), 629-644.

Van Laar, D, Edwards, J., \& Easton, S. (2007). The workrelated quality of life scale for healthcare workers. Journal of Advanced Nursing, 60 (3), 325-333.

Veenhoven, R. (1996). Happy life expectancy: a comprehensive measure of quality-of-life in nations. Social Indicators Research, 39 (1), 1-58.

Warr, P. (2007). Work, happiness and unhappiness. Mahwah, NJ: LEA.

WHOQOL Group. (1994). The development of the World Health Organization quality of life assessment instrument. In J. Orley, W. Kuyken. Quality oflife assessment: international perspectives (pp.41-60). Heidelberg: Springer Verlag.

Recebido em: 14/4/2011

Versão final em: 19/8/2011

Aprovado em: 13/3/2012 
